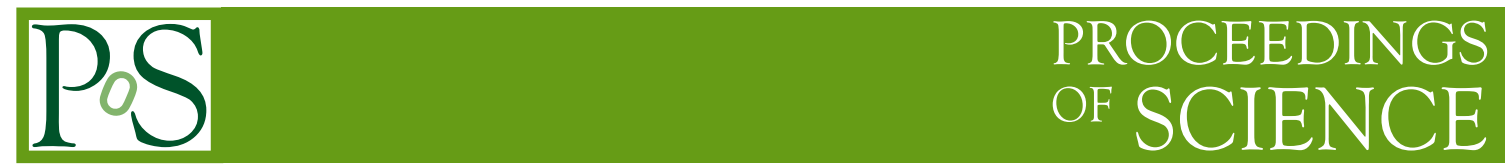

\title{
The rare decay $H \rightarrow Z \gamma$ in perturbative QCD
}

\section{Dominik Kara*}

Physik-Institut, Universität Zürich, Winterthurerstrasse 190, 8057 Zürich, Switzerland

E-mail: dkara@physik.uzh.ch

The rare Higgs boson decay $H \rightarrow Z \gamma$ is forbidden at tree-level. In the Standard Model, it is loopmediated through a $W$ boson or a heavy quark. We analytically compute the QCD correction to the heavy quark loop, confirming earlier purely numerical results, that were obtained for on-shell renormalization. We investigate the numerical interplay of bottom and top quark contributions, and the dependence of the result on the renormalization scheme.

12th International Symposium on Radiative Corrections (Radcor 2015) and LoopFest XIV (Radiative Corrections for the LHC and Future Colliders)

15-19 June 2015

UCLA Department of Physics \& Astronomy Los Angeles, CA, USA

${ }^{*}$ Speaker. 


\section{Introduction}

The $H \rightarrow \gamma \gamma$ decay mode was among the most significant signatures in the Higgs boson discovery $[1,2]$, it has been measured in the meantime $[3,4]$ with a relative precision of below twenty per cent. The branching ratio for $H \rightarrow Z \gamma$, including the leptonic branching ratio of the $Z$ boson, is considerably lower and only upper bounds could be established on it up to now [5, 6]. Once established, this decay will provide access to a broader spectrum of observables than $H \rightarrow \gamma \gamma$, since the decay of the $Z$ boson to leptons will enable the study of spin-dependent particle correlations.

Higher order QCD corrections to $H \rightarrow Z \gamma$ from gluon exchange in the top quark loop were derived in [7] by performing a purely numerical evaluation of the relevant two-loop integrals in terms of five-dimensional Feynman parameter representations. The results derived in [7] use an on-shell renormalization for the top quark mass and the Yukawa coupling. In this talk, we report on the analytical computation of the NLO QCD corrections to $H \rightarrow Z \gamma$ as described in [8].

Besides its phenomenological implications for $H \rightarrow Z \gamma$, our calculation also provides an important subset of two-loop integrals relevant to the two-loop amplitudes $g g \rightarrow H g$ and $q g \rightarrow H q$ with full top quark mass dependence. These amplitudes are known at present only at one loop [9], corresponding to the leading order in perturbation theory. For precision studies of the transverse momentum distribution of the Higgs boson and of Higgs-boson-plus-jet production, an effective field theory in the limit of infinite top quark mass is used commonly. In this approach, NLO QCD corrections were derived [10-13] and the calculation of the NNLO corrections is welladvanced [14-18]. The effective field theory description is however inappropriate at large transverse momenta, where the top quark loop is resolved by the recoiling jet; and it is precisely in this region that deviations from the Standard Model due to new heavy states could become visible. The calculation of NLO QCD corrections with exact top quark mass dependence is therefore recognized as high-priority aim $[19,20]$, and the integrals derived here will be an important step towards it.

\section{The $H \rightarrow Z \gamma$ decay in the Standard Model}

The decay width $H \rightarrow Z \gamma$ is obtained as

$$
\Gamma=\frac{G_{F}^{2} \alpha m_{W}^{2}}{4 m_{H}^{3}\left(m_{H}^{2}-m_{Z}^{2}\right)}|A|^{2}
$$

with Fermi's coupling constant $G_{F}$, the fine-structure constant $\alpha$ and the Higgs and $Z$ boson masses $m_{H}$ and $m_{Z}$, respectively. Depending on the particle coupled to the external Higgs boson, the amplitude can be further decomposed into contributions from the $W$ boson and the fermions $q$ :

$$
A=c_{W} A_{W}+\sum_{q} c_{q} A_{q}
$$

The coupling factors are

$$
c_{W}=\cos \theta_{w}, \quad c_{q}=N_{c} \frac{2 Q_{q}\left(I_{q}^{3}-2 Q_{q} \sin ^{2} \theta_{w}\right)}{\cos \theta_{w}},
$$

where $\theta_{w}$ is the weak mixing angle, $N_{c}$ the number of colors, $Q_{q}$ the charge of the fermion and $I_{q}^{3}$ the third component of its weak isospin. Due to the mass hierarchy of the particles involved, we 
will only consider the dominant pieces coming from the $W$ boson, the top quark and the bottom quark.

The Born-level contribution to the amplitude arises at one loop, it is written as:

$$
A^{(1)}=c_{W} A_{W}^{(1)}+c_{t} A_{t}^{(1)}+c_{b} A_{b}^{(1)} .
$$

Higher-order perturbative corrections are obtained by a loop expansion of the amplitude $A$. Nextto-leading order QCD corrections affect only $A_{t}$ and $A_{b}$, they correspond to two-loop graphs with an internal mass:

$$
A_{q}\left(m_{H}, m_{Z}, m_{q}, \alpha_{s}, \mu\right)=A_{q}^{(1)}\left(m_{H}, m_{Z}, m_{q}\right)+\frac{\alpha_{s}(\mu)}{\pi} A_{q}^{(2)}\left(m_{H}, m_{Z}, m_{q}, \mu\right) .
$$

\section{Calculation of the two-loop amplitude}

To compute the two-loop QCD contribution $A_{q}^{(2)}$, we project all Feynman diagrams generated by QGRAF [21] onto the relevant tensor structure using FORM [22]. The resulting Feynman integrals are reduced to a set of master integrals with the help of integration-by-parts (IBP) identities [23], which are solved using the Laporta algorithm [24] implemented in the REDUZE2 code $[25,26]$. After the reduction, the amplitude can be expressed in terms of a certain number of master integrals depending on the loop order.

Each of these master integrals has a specific mass dimension, which can be scaled out by multiplying with the appropriate power of the mass $m_{q}$ running in the loop, such that the resulting dimensionless integrals are only functions of the mass ratios $m_{H}^{2} / m_{q}^{2}$ and $m_{Z}^{2} / m_{q}^{2}$. We parametrize this dependence by introducing Landau-type variables

$$
m_{H}^{2}=-m_{q}^{2} \frac{(1-h)^{2}}{h}, \quad m_{Z}^{2}=-m_{q}^{2} \frac{(1-z)^{2}}{z} .
$$

\subsection{Differential equations and integral basis}

To compute the two-loop master integrals, we use the method of differential equations [2730]. In this method, differential equations in internal masses and external invariants are derived for each integral by performing the differentiation on the integrand, which is then related to the original master integral by the IBP identities. With this, we obtain inhomogeneous differential equations in either Landau variable, plus a trivial homogeneous equation in $m_{q}$ for each integral. The differential equations are solved in a bottom-up approach, i.e. starting from the master integrals with the lowest number of different propagators ('topology') because they will show up in differential equations of higher topologies.

The coefficients of the individual master integrals in the homogeneous and inhomogeneous terms of the differential equations are rational functions of $h$ and $z$. Upon partial fractioning, only a limited number of polynomials in $h$ and $z$ appear. These form the so-called alphabet $\left\{l_{1}, \ldots, l_{12}\right\}$ associated with this set of master integrals:

$$
\begin{array}{llll}
l_{1}=z, & l_{4}=h, & l_{7}=h-z, & l_{10}=h^{2} z-h z-h+z, \\
l_{2}=z+1, & l_{5}=h+1, & l_{8}=h z-1, & l_{11}=z^{2}-h z-z+1, \\
l_{3}=z-1, & l_{6}=h-1, & l_{9}=h^{2}-h z-h+1, & l_{12}=z^{2} h-h z-z+h .
\end{array}
$$


With an appropriate choice of basis integrals [30], the full system of differential equations for all 28 master integrals (written as 28-component vector $\vec{M}$ ) takes the form of a total differential,

$$
\mathrm{d} \vec{M}(h, z)=\varepsilon \sum_{k=1}^{12} R_{k} \mathrm{~d} \log \left(l_{k}\right) \vec{M}(h, z),
$$

where the matrices $R_{k}$ contain only rational numbers. In this case, two important features can be exploited. First, the differential equations can be integrated order by order in $\varepsilon$ in terms of generalized harmonic polylogarithms (GHPLs) [31-34]. They are defined as iterated integrals according to

$$
G\left(w_{1}, \ldots, w_{n} ; x\right) \equiv \int_{0}^{x} \mathrm{~d} t \frac{1}{t-w_{1}} G\left(w_{2}, \ldots, w_{n} ; t\right), \quad G\left(\overrightarrow{0}_{n} ; x\right) \equiv \frac{\log ^{n} x}{n !},
$$

with indices $w_{i}$ and argument $x$. Second, the results will be expressed as a linear combination of GHPLs of homogeneous weight. We arrive at a total differential of the form (3.3) starting from the Laporta basis $\vec{I}$ depicted in Fig. 1 and subsequently applying the algorithm described in [35].

Since the alphabet (3.2) is not linear in the Landau variables, we further decompose it to enable the integration in either $h$ or $z$, which yields a solution up to an integration constant that only depends on the other variable. This boundary value is then determined by imposing regularity in special kinematic points, where the integrals are known to be regular from physical arguments. In our case, these points are given by

$$
\begin{aligned}
& h=1 \quad \leftrightarrow \quad m_{H}^{2}=0, \quad h=z \quad \leftrightarrow \quad m_{H}^{2}=m_{Z}^{2}, \\
& z=1 \quad \leftrightarrow \quad m_{Z}^{2}=0, \quad h=\frac{1}{z} \quad \leftrightarrow \quad m_{H}^{2}=m_{Z}^{2},
\end{aligned}
$$

i.e. they correspond to the limit where the masses of the external particles either vanish or coincide.

By taking limits in these kinematic points, we are left with GHPLs that contain the same variable $x \in\{h, z\}$ both in the argument and in the indices. In order to simplify the result and to obtain a unique representation, we use an inhouse MATHEMATICA implementation [36, 37] relying on the symbol and coproduct formalism [38] to transform GHPLs of the type

$$
G\left(w_{1}(x), \ldots, w_{n}(x) ; x\right) \rightarrow G\left(a_{1}, \ldots, a_{n} ; x\right),
$$

where the transformed indices $a_{i}$ are nothing but complex numbers. In doing so, we end up with GHPLs up to weight four, which are given by

$$
\begin{aligned}
& G\left(a_{1}, \ldots, a_{n} ; h\right) \quad \text { with } \quad a_{i} \in\left\{0, \pm 1, z, \frac{1}{z}, J_{z}, \frac{1}{J_{z}}, K_{z}^{ \pm}, L_{z}^{ \pm}\right\} \\
& G\left(b_{1}, \ldots, b_{n} ; z\right) \quad \text { with } \quad b_{i} \in\{0, \pm 1, c, \bar{c}\}
\end{aligned}
$$

where

$$
\begin{aligned}
c & =\frac{1}{2}(1+i \sqrt{3}), & K_{z}^{ \pm} & =\frac{1}{2}\left(1+z \pm \sqrt{-3+2 z+z^{2}}\right), \\
J_{z} & =\frac{z}{1-z+z^{2}}, & L_{z}^{ \pm} & =\frac{1}{2 z}\left(1+z \pm \sqrt{1+2 z-3 z^{2}}\right)
\end{aligned}
$$




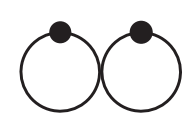

$I_{1}$

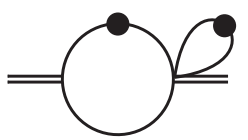

$I_{5}$

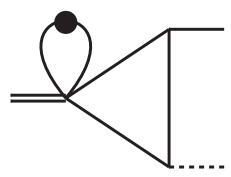

$I_{9}$

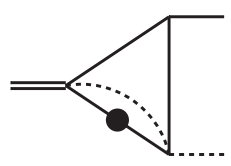

$I_{13}$

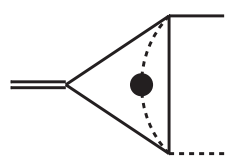

$I_{17}$

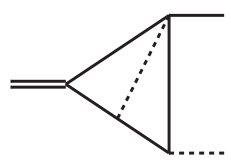

$I_{21}$

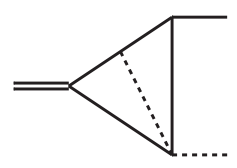

$I_{25}$

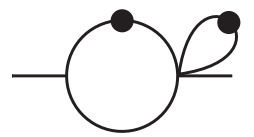

$I_{2}$

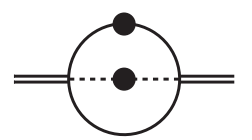

$I_{6}$

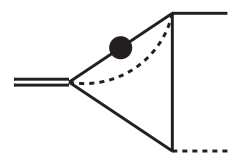

$I_{10}$

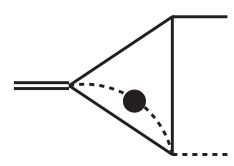

$I_{14}$

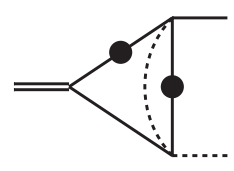

$I_{18}$
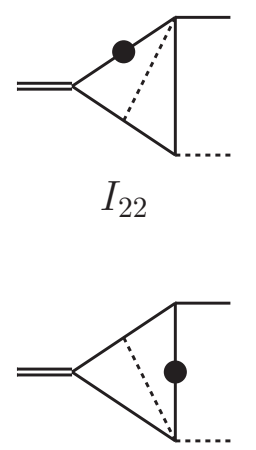

$I_{26}$

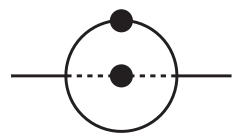

$I_{3}$

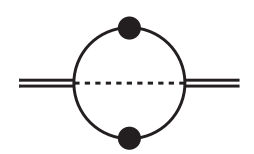

$I_{7}$

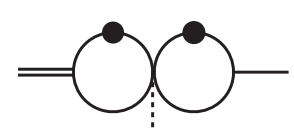

$I_{11}$

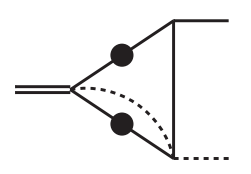

$I_{15}$

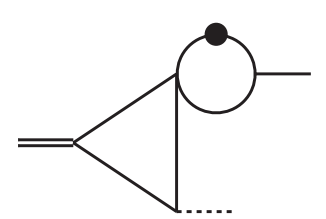

$I_{19}$

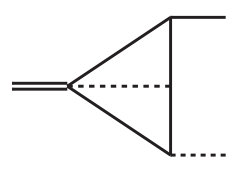

$I_{23}$

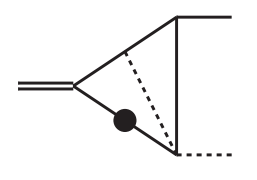

$I_{27}$

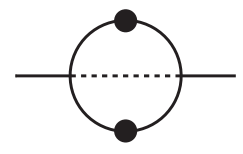

$I_{4}$

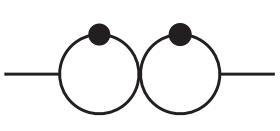

$I_{8}$

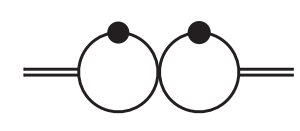

$I_{12}$
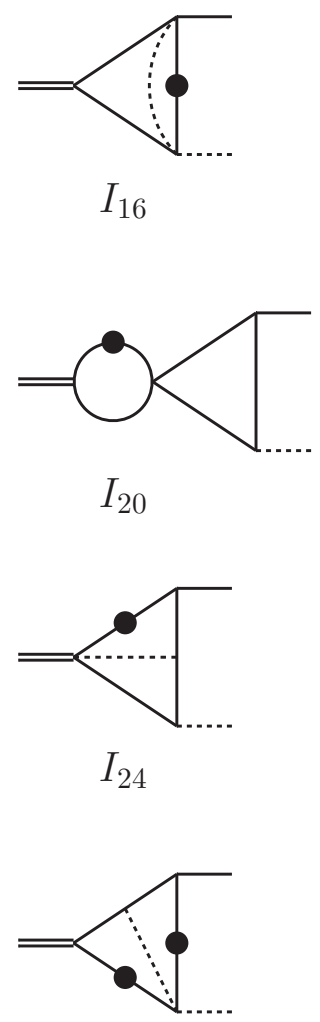

$I_{28}$

Figure 1: Two-loop master integrals for the calculation of $A_{q}^{(2)}$. Dashed lines are massless, whereas internal solid lines denote propagators with mass $m_{q}$. Double and solid external lines correspond to virtualities $m_{H}^{2}$ and $m_{Z}^{2}$, respectively. Dotted propagators are taken to be squared. 
for the underlying set of master integrals. Further transformations of the type

$$
G\left(w_{1}(x), \ldots, w_{n}(x) ; y\right) \rightarrow G\left(c_{1}(y), \ldots, c_{n}(y) ; x\right)
$$

become necessary when the integration is performed in a different variable compared to the integration of a master integral of a subtopology which enters the differential equation under consideration.

We would like to state that the results of the master integrals were checked in several ways. We performed transformations of the type (3.9) and verified that the solution fulfills the differential equation in the other variable. This check works only up to a constant, which is why we compared each master integral numerically against SECDEC [39] and found agreement to high precision.

\subsection{Renormalization}

After the manipulations described in the beginning of Section 3 and after inserting the analytic results of the master integrals, we are left with the unrenormalized two-loop amplitude.

For its renormalization, we consider three different prescriptions:

(a) quark mass and Yukawa coupling in the on-shell (OS) scheme.

(b) quark mass in the OS scheme, Yukawa coupling in the $\overline{\mathrm{MS}}$ scheme.

(c) quark mass and Yukawa coupling in the $\overline{\mathrm{MS}}$ scheme.

All three prescriptions yield the same pole parts of the renormalization counter terms and produce finite expressions for the renormalized amplitude. They are related by finite scheme transformations, which is why we choose to compute the renormalized amplitude in scheme (a) and use it to derive the results in schemes (b) and (c). In the pure OS scheme, the quantity

$$
\frac{1}{m_{q}} \delta m_{\mathrm{OS}} C_{q}^{(1)}+Z_{\mathrm{OS}} A_{q}^{(1)}
$$

has to be added to the unrenormalized two-loop amplitude in order to remove its divergences, where $Z_{\mathrm{OS}}$ and $\delta m_{\mathrm{OS}}$ are the Yukawa and mass renormalization constants, respectively [40]:

$$
Z_{\mathrm{OS}}=\frac{\alpha_{s}(\mu)}{\pi} 16 i \pi^{2} S_{\varepsilon} \frac{C_{F}}{4} \frac{3-2 \varepsilon}{\varepsilon(1-2 \varepsilon)}, \quad \delta m_{\mathrm{OS}}=m_{q} Z_{\mathrm{OS}} .
$$

Next, we express the OS quantities $M_{q}$ and $Y_{q}$ in terms of $\overline{\mathrm{MS}}$ quantities $\bar{m}_{q}$ and $\bar{y}_{q}$ at a particular matching scale $\mu_{m}$ using the standard relations (e.g. [41-43]):

$$
M_{q}=\bar{m}_{q}(\mu)(1+\Delta), \quad Y_{q}=\bar{y}_{q}(\mu)(1+\Delta), \quad \Delta=\frac{\alpha_{s}(\mu)}{\pi} C_{F}\left(1+\frac{3}{4} \log \frac{\mu^{2}}{\bar{m}_{q}^{2}(\mu)}\right) .
$$

We perform this matching at the scale of the running $\overline{\mathrm{MS}}$ quark mass $\bar{m}_{q}$. Starting from the OS result $A_{q}^{(2, a)}\left(m_{H}, m_{Z}, M_{q}\right)$, these scheme transformations induce finite shifts in the amplitudes of the prescriptions (b) and (c):

$$
\begin{aligned}
& A_{q}^{(2, b)}\left(m_{H}, m_{Z}, \bar{m}_{q}, \mu\right)=A_{q}^{(2, a)}\left(m_{H}, m_{Z}, \bar{m}_{q}(\mu)\right)+\Delta \cdot A_{q}^{(1)}\left(m_{H}, m_{Z}, \bar{m}_{q}(\mu)\right) \\
& A_{q}^{(2, c)}\left(m_{H}, m_{Z}, \bar{m}_{q}, \mu\right)=A_{q}^{(2, b)}\left(m_{H}, m_{Z}, \bar{m}_{q}(\mu)\right)+\left.\bar{\Delta} \cdot \frac{\partial A_{q}^{(1)}\left(m_{H}, m_{Z}, M_{q}\right)}{\partial M_{q}}\right|_{M_{q}=\bar{m}_{q}(\mu)}
\end{aligned}
$$


In practice, the coefficient $\bar{\Delta}$ emerges by making the following replacements in $A_{q}^{(1)}$, where the Landau variables $\bar{h}$ and $\bar{z}$ are defined according to Eq. (3.1) with $m_{q}=\bar{m}_{q}(\mu)$ :

$$
h=\bar{h}-2 \Delta \bar{h} \frac{\bar{h}-1}{\bar{h}+1}, \quad z=\bar{z}-2 \Delta \bar{z} \frac{\bar{z}-1}{\bar{z}+1} .
$$

\section{Numerical results}

The calculation of the master integrals and the amplitude outlined in Section 3 is performed in the Euclidean region, where $m_{H}^{2}$ and $m_{Z}^{2}$ in Eq. (3.1) are negative and the master integrals are real. In order to get a physical expression, the results have to be analytically continued to the physical Minkowski region, where we distinguish three kinematic regions:

$$
\text { I: } \quad m_{Z}^{2}<m_{H}^{2}<4 m_{q}^{2}, \quad \text { II: } \quad m_{Z}^{2}<4 m_{q}^{2}<m_{H}^{2}, \quad \text { III: } 4 m_{q}^{2}<m_{Z}^{2}<m_{H}^{2} \text {. }
$$

It is obvious that the top quark amplitude is calculated in Region I, while the bottom quark amplitude is computed in Region III. Region II is not needed for the physical values of the masses.

Since the analytical expression for $A_{q}^{(2)}$ is given in terms of GHPLs, it can be evaluated using GINAC $[34,44]$. This leads to the following next-to-leading-order decay width $\Gamma^{(2)}$ in the renormalization schemes (a), (b) and (c):

$$
\begin{aligned}
\Gamma^{(2, a)} & =\left[7.07533+0.42800 \frac{\alpha_{s}(\mu)}{\pi}\right] \mathrm{keV} \\
\stackrel{\mu}{\mu}=m_{H} & \stackrel{=}{=} .09072 \mathrm{keV} \\
\Gamma^{(2, b)} \stackrel{\mu}{ } & \stackrel{=}{=}\left[7.09409+\frac{\alpha_{s}\left(m_{H}\right)}{\pi}\left(-0.53266-0.76661 \log \frac{m_{H}^{2}}{\bar{m}_{t}^{2}\left(m_{H}\right)}+0.01229 \log \frac{m_{H}^{2}}{\bar{m}_{b}^{2}\left(m_{H}\right)}\right)\right] \mathrm{keV} \\
& =7.09403 \mathrm{keV} \\
\Gamma^{(2, c)} \stackrel{\mu}{ } & \stackrel{=}{=}\left[7.05934+\frac{\alpha_{s}\left(m_{H}\right)}{\pi}\left(0.64587+0.10597 \log \frac{m_{H}^{2}}{\bar{m}_{t}^{2}\left(m_{H}\right)}+0.01453 \log \frac{m_{H}^{2}}{\bar{m}_{b}^{2}\left(m_{H}\right)}\right)\right] \mathrm{keV} \\
& =7.08438 \mathrm{keV} .
\end{aligned}
$$

An estimation of the uncertainty on the prediction from missing higher orders is provided by varying $m_{H} / 2<\mu<2 m_{H}$ in Fig. 2.

We observe that the NLO results for the decay width are consistent between the three schemes. The relative size of the NLO correction is $2 \%$ in scheme (a), below $10^{-5}$ in scheme (b) and $3 \%$ in scheme (c). The very small corrections in scheme (b) are however in large part due to numerical cancellations between a priori unrelated terms. The spread between the different schemes is $1.3 \%$ at $\mu=m_{H}$, and variations of the renormalization scale change the predictions in either given scheme by at most $0.4 \%$.

\section{Conclusions}

We have revisited the QCD corrections to the rare loop-induced Higgs boson decay $H \rightarrow Z \gamma$. The relevant two-loop three-point integrals with two different external masses and one internal mass were derived analytically, using a reduction to master integrals, which were then computed using 


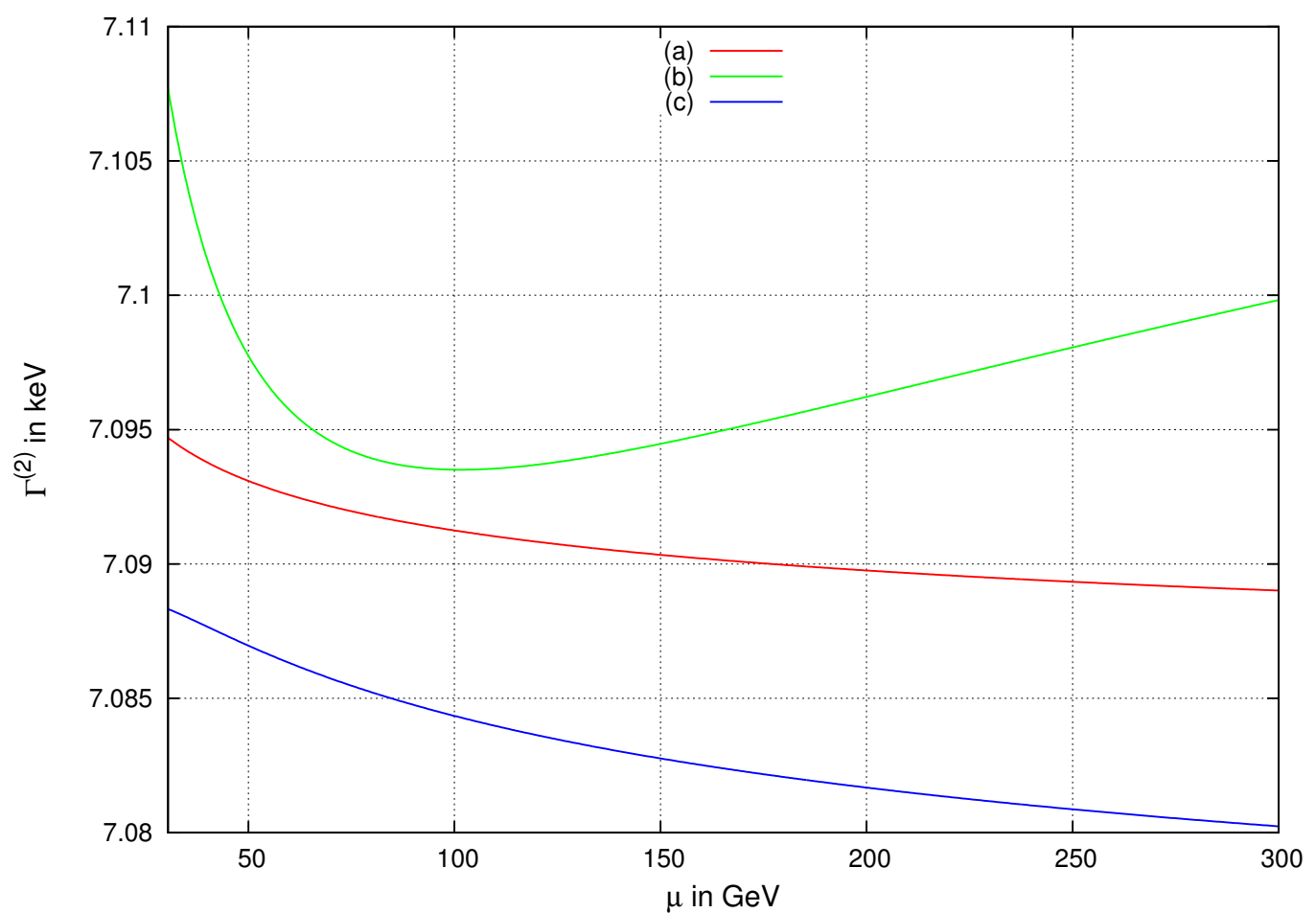

Figure 2: Scale variation of the next-to-leading-order decay width $\Gamma^{(2)}$ in the renormalization schemes (a), (b) and (c) for $30 \mathrm{GeV}<\mu<300 \mathrm{GeV}$.

differential equations. These integrals are also an important ingredient to the two-loop amplitudes for Higgs-plus-jet production in gluon fusion with full dependence on the internal quark masses.

We investigated the dependence of the corrections on the renormalization scheme used for the quark mass and Yukawa coupling. We observe that the results for the decay rate in on-shell and $\overline{\mathrm{MS}}$ schemes, as well as in a hybrid scheme with on-shell mass and $\overline{\mathrm{MS}}$ Yukawa coupling, are well consistent with each other, and that corrections are in the sub-per-cent range in all three schemes (being smallest in the hybrid scheme). We confirm the previously available numerical on-shell result [7] and agree with an independent caclulation [? ]. The residual QCD uncertainty on the $H \rightarrow Z \gamma$ decay rate is around $1.7 \%$ o from the combination of scale variation and spread between the different renormalization schemes.

\section{References}

[1] G. Aad et al. [ATLAS Collaboration], Phys. Lett. B 716 (2012) 1 [arXiv:1207.7214 [hep-ex]].

[2] S. Chatrchyan et al. [CMS Collaboration], Phys. Lett. B 716 (2012) 30 [arXiv:1207.7235 [hep-ex]].

[3] G. Aad et al. [ATLAS Collaboration], Phys. Rev. D 90 (2014) 11, 112015 [arXiv:1408.7084 [hep-ex]]. 
[4] V. Khachatryan et al. [CMS Collaboration], Eur. Phys. J. C 74 (2014) 10, 3076 [arXiv:1407.0558 [hep-ex]].

[5] G. Aad et al. [ATLAS Collaboration], Phys. Lett. B 732 (2014) 8 [arXiv:1402.3051 [hep-ex]].

[6] S. Chatrchyan et al. [CMS Collaboration], Phys. Lett. B 726 (2013) 587 [arXiv:1307.5515 [hep-ex]].

[7] M. Spira, A. Djouadi and P. M. Zerwas, Phys. Lett. B 276 (1992) 350.

[8] T. Gehrmann, S. Guns and D. Kara, JHEP 1509 (2015) 038 [arXiv:1505.00561 [hep-ph]].

[9] M. Spira, A. Djouadi, D. Graudenz and P. M. Zerwas, Nucl. Phys. B 453 (1995) 17 [hep$\mathrm{ph} / 9504378]$.

[10] C. R. Schmidt, Phys. Lett. B 413 (1997) 391 [hep-ph/9707448].

[11] C. J. Glosser and C. R. Schmidt, JHEP 0212 (2002) 016 [hep-ph/0209248].

[12] D. de Florian, M. Grazzini and Z. Kunszt, Phys. Rev. Lett. 82 (1999) 5209 [hep-ph/9902483].

[13] V. Ravindran, J. Smith and W. L. Van Neerven, Nucl. Phys. B 634 (2002) 247 [hep$\mathrm{ph} / 0201114]$.

[14] T. Gehrmann, M. Jaquier, E. W. N. Glover and A. Koukoutsakis, JHEP 1202 (2012) 056 [arXiv:1112.3554 [hep-ph]].

[15] R. Boughezal, F. Caola, K. Melnikov, F. Petriello and M. Schulze, JHEP 1306 (2013) 072 [arXiv:1302.6216 [hep-ph]].

[16] X. Chen, T. Gehrmann, E. W. N. Glover and M. Jaquier, Phys. Lett. B 740 (2015) 147 [arXiv:1408.5325 [hep-ph]].

[17] R. Boughezal, F. Caola, K. Melnikov, F. Petriello and M. Schulze, Phys. Rev. Lett. 115 (2015) 8, 082003 [arXiv:1504.07922 [hep-ph]].

[18] R. Boughezal, C. Focke, W. Giele, X. Liu and F. Petriello, Phys. Lett. B 748 (2015) 5 [arXiv:1505.03893 [hep-ph]].

[19] S. Dittmaier et al. [LHC Higgs Cross Section Working Group], Handbook of LHC Higgs Cross Sections: 2. Differential Distributions, arXiv:1201.3084 [hep-ph].

[20] S. Heinemeyer et al. [LHC Higgs Cross Section Working Group], Handbook of LHC Higgs Cross Sections: 3. Higgs Properties, arXiv:1307.1347 [hep-ph].

[21] P. Nogueira, J. Comput. Phys. 105 (1993) 279.

[22] J. Kuipers, T. Ueda, J. A. M. Vermaseren and J. Vollinga, Comput. Phys. Commun. 184 (2013) 1453 [arXiv:1203.6543 [cs.SC]].

[23] K. G. Chetyrkin and F. V. Tkachov, Nucl. Phys. B 192 (1981) 159. 
[24] S. Laporta, Int. J. Mod. Phys. A 15 (2000) 5087 [hep-ph/0102033].

[25] A. von Manteuffel and C. Studerus, arXiv:1201.4330 [hep-ph].

[26] R. Lewis, Computer Algebra System Fermat, http://www.bway.net/ lewis.

[27] A. V. Kotikov, Phys. Lett. B 254 (1991) 158.

[28] E. Remiddi, Nuovo Cim. A 110 (1997) 1435 [hep-th/9711188].

[29] T. Gehrmann and E. Remiddi, Nucl. Phys. B 580 (2000) 485 [hep-ph/9912329].

[30] J. M. Henn, Phys. Rev. Lett. 110 (2013) 25, 251601 [arXiv:1304.1806 [hep-th]].

[31] A. B. Goncharov, Math. Res. Lett. 5 (1998) 497 [arXiv:1105.2076 [math.AG]].

[32] E. Remiddi and J. A. M. Vermaseren, Int. J. Mod. Phys. A 15 (2000) 725 [hep-ph/9905237].

[33] T. Gehrmann and E. Remiddi, Nucl. Phys. B 601 (2001) 248 [hep-ph/0008287].

[34] J. Vollinga and S. Weinzierl, Comput. Phys. Commun. 167 (2005) 177 [hep-ph/0410259].

[35] T. Gehrmann, A. von Manteuffel, L. Tancredi and E. Weihs, JHEP 1406, 032 (2014) [arXiv:1404.4853 [hep-ph]].

[36] T. Gehrmann, L. Tancredi and E. Weihs, JHEP 1308 (2013) 070 [arXiv:1306.6344 [hep-ph]].

[37] E. Weihs, Dark Higgs studies and diboson production at the Large Hadron Collider, Dissertation Universität Zürich (2013).

[38] C. Duhr, JHEP 1208 (2012) 043 [arXiv:1203.0454 [hep-ph]].

[39] S. Borowka, G. Heinrich, S. P. Jones, M. Kerner, J. Schlenk and T. Zirke, arXiv:1502.06595 [hep-ph].

[40] W. Bernreuther, R. Bonciani, T. Gehrmann, R. Heinesch, T. Leineweber, P. Mastrolia and E. Remiddi, Nucl. Phys. B 706 (2005) 245 [hep-ph/0406046].

[41] K. Melnikov and T. v. Ritbergen, Phys. Lett. B 482 (2000) 99 [hep-ph/9912391].

[42] K. G. Chetyrkin and M. Steinhauser, Nucl. Phys. B 573 (2000) 617 [hep-ph/9911434].

[43] M. Jamin and A. Pich, Nucl. Phys. B 507 (1997) 334 [hep-ph/9702276].

[44] C. W. Bauer, A. Frink and R. Kreckel, J.Symb.Comput. 33 (2002) 1 [cs/0004015 [cs-sc]].

[45] R. Bonciani, V. Del Duca, H. Frellesvig, J. M. Henn, F. Moriello and V. A. Smirnov, JHEP 1508 (2015) 108 [arXiv:1505.00567 [hep-ph]]. 